\title{
Computational Modeling and Experiments of Natural Convection for a Titan Montgolfiere
}

\author{
Tim Colonius* and Daniel Appelö ${ }^{\dagger}$ \\ California Institute of Technology, Pasadena, CA USA \\ Julian Nott $\ddagger$ \\ Nott Technology LLC, Santa Barbara, CA, USA \\ Jeffrey Hall ${ }^{\S}$ \\ Jet Propulsion Laboratory, Pasadena, CA, USA
}

\begin{abstract}
Computational models are developed to predict the natural convection heat transfer and buoyancy for a Montgolfiere under conditions relevant to the Titan atmosphere. Idealized single and double-walled balloon geometries are simulated using algorithms suitable for both laminar and (averaged) turbulent convection. Steady-state performance results are compared to existing heat transfer coefficient correlations. The laminar results, in particular, are used to test the validity of the correlations in the absence of uncertainties associated with turbulence modeling. Some discrepancies are observed, especially for convection in the gap, and appear to be primarily associated with temperature nonuniformity on the balloon surface. The predicted buoyancy for the single-walled balloon in the turbulent convection regime, predicted with a standard $k-\epsilon$ turbulence model, was within $10 \%$ of predictions based on the empirical correlations. There was also good agreement with recently conducted experiments in a cryogenic facility designed to simulate the Titan atmosphere.
\end{abstract}

\section{Nomenclature}

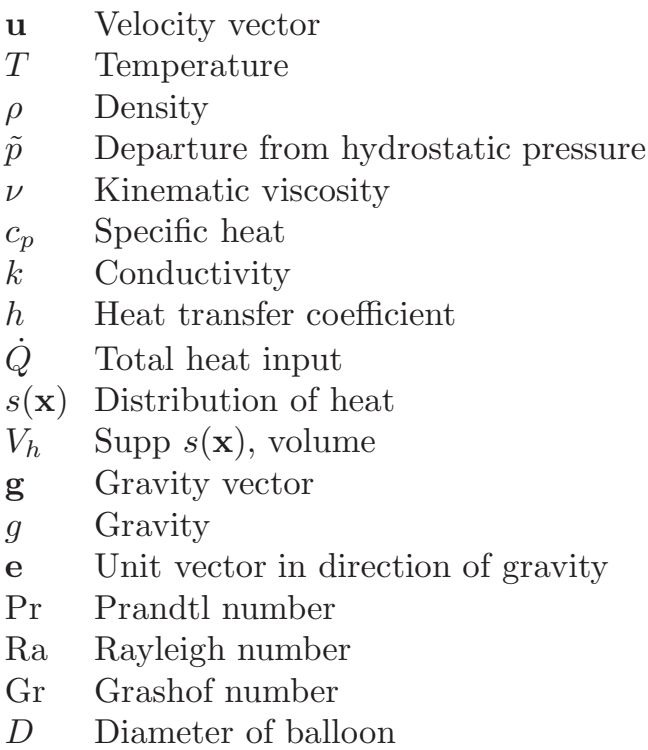

*Professor, Division of Engineering and Applied Science, AIAA Senior member

${ }^{\dagger}$ Postdoctoral Scholar, Applied and Computational Math

¥President, AIAA Senior member

${ }^{\S}$ Senior Engineer, Mobility and Robotic Systems, AIAA Senior Member 
$\phi \quad$ Ratio of inner and outer diameter

$\Delta T$ Temperature differences with ambient temperature

$\Delta T_{b}$ Average temperature within the balloon

$\Delta T_{s}$ Average surface temperature

$\beta \quad$ Coefficient of thermal expansion

$\frac{D}{D t} \quad$ Material Derivative

Subscript

$\infty \quad$ Ambient value

$i \quad$ Inner

$o \quad$ Outer

\section{Introduction}

Saturn's moon Titan holds immense scientific interest, and a Montgolfiere, or hot air balloon, is an attractive aerobot configuration for its exploration. The cold, dense atmosphere of Titan, with gravity about one-seventh that of Earth, requires a significantly reduced heat input for a given balloon mass, compared to Earth. The smaller heat input also implies that natural convection, rather than radiation, will dominate the heat loss to the environment. For example, estimates utilizing heat transfer correlations show that as little as $2 \mathrm{KW}$ of power may be sufficient for certain scientific missions. However, more accurate predictions of turbulent convection are sought in order to carefully establish mission feasibility, uncertainty, and safety factors. The present paper reports on efforts to construct a detailed computational fluid dynamic/thermal model for this purpose.

Existing natural heat transfer correlations, described in detail in Section II, are very useful for system-level balloon models, ${ }^{1}$ but strictly apply only to simplified model problems such as the natural convection around a constant-temperature (or uniformly heated) sphere. Their application to hot air balloons, where the heat source and or temperature fields are nonuniform, involves uncertainties that have not yet been assessed. Moreover, heat transfer correlations depend on whether the induced convective flow is laminar or turbulent. While the scale of the proposed Titan Montgolfiere implies that natural convection will be turbulent, it is of interest to examine the correlations over a wide range of heat source strengths. The reason for this is that for the turbulent case, the computational models themselves involve turbulence models with adjustable parameters, and whose uncertainty is not known a priori. For the laminar case, by contrast, any difference between the computational model and the heat transfer correlations can be attributed to the non-uniformity of temperature and heat flux at the balloon surfaces.

Thus, we consider laminar and turbulent natural convection around balloons with idealized heat sources, including single and double-walled balloons

Figure 1. Schematic of single-walled JPL Titan Montgolfiere. with spherical and sphere-on-cone geometries. The full governing equations describing conservation of mass, momentum, and energy are solved internal and external to the balloon membrane(s). Estimates show that under relevant conditions, a Boussinesq approximation can be used, wherein the fluid is assumed nearly incompressible and the buoyancy force is proportional to temperature fluctuations. The flow is idealized as axisymmetric in order to reduce computational effort. To simplify the modeling we initially restrict our 
attention to steady-state performance at fixed altitude. For the turbulent cases, we utilize a Reynoldsaveraged-Navier-Stokes (RANS) turbulence model. From the computed results, flow patterns are examined and steady-state temperature fields are analyzed to determine the buoyancy (lift force), as a function of heat input, and to compare to existing (semi-empirical) heat transfer correlations. For the turbulent convection case, we validate the computational model by comparing the results with preliminary experiments performed in the Titan Sky Simulator ${ }^{\text {TM2 }}$ facility designed to assess the buoyancy of Titan Montgolfiere prototypes under simulated cryogenic conditions comparable to the Titan atmosphere.

The remainder of the paper is organized as follows. In the next section, we review the relevant existing heat transfer correlations for natural convection internal and external to heated spheres and spherical annuli. In Sections III and IV we present the computational and experimental methodology, respectively. In Section $V$ we present the results of laminar simulations for spherical, double-walled balloons, and assess the accuracy of the individual heat transfer correlations, internal and external to the balloon, and in the gap. In Section VI, we present results for the turbulent convection case, and compare our results to the aforementioned experimental results. Sensitivity of the computed results to model parameters is assessed for the turbulent case, and briefly summarized in an appendix. The paper concludes with a brief summary of findings in Section VII.

\section{Previous theory and correlations for natural convection heat transfer}

\section{A. Boussinesq flow model}

In a typical balloon, the temperature difference between the balloon and ambient air, $T-T_{\infty}$, is small compared to the ambient temperature. Thus it is appropriate to use the incompressible Boussinesq flow model:

$$
\begin{aligned}
\nabla \cdot \mathbf{u} & =0 \\
\rho_{\infty} \frac{D \mathbf{u}}{D t}+\nabla \tilde{p} & =-\rho_{\infty} \frac{T-T_{\infty}}{T_{\infty}} \mathbf{g}+\mu_{\infty} \nabla^{2} \mathbf{u} \\
\rho_{\infty} c_{p} \frac{D T}{D t} & =k_{\infty} \nabla^{2} T+\frac{\dot{Q}}{V_{h}} s(\mathbf{x}) .
\end{aligned}
$$

Note that we have neglected the temperature dependence of $k$ and $\mu$; this is valid to the same order as the Boussinesqu approximation. Let

$$
\tilde{\theta}=\frac{g D^{3}}{\nu_{\infty}^{2}} \theta=\frac{g D^{3}}{\nu_{\infty}^{2}} \frac{T-T_{\infty}}{T_{\infty}}
$$

Then the equations may be rewritten in the non-dimensional form

$$
\begin{aligned}
\nabla \cdot \mathbf{u} & =0, \\
\frac{D \mathbf{u}}{D t}+\nabla \tilde{p} & =-\tilde{\theta} \mathbf{e}+\nabla^{2} \mathbf{u}, \\
\frac{D \tilde{\theta}}{D t} & =\frac{1}{\operatorname{Pr}} \nabla^{2} \tilde{\theta}+\tilde{Q} \frac{D^{3}}{V_{h}} s(\mathbf{X}),
\end{aligned}
$$

with the new parameter

$$
\tilde{Q}=\frac{g D^{2} \dot{Q}}{\rho c_{p} T_{\infty} \nu_{\infty}^{3}}
$$

From these equations we may surmise that all the solutions to the problem may be determined by specifying just two non-dimensional parameters, the Prandtl number and the non-dimensional heat input, $\tilde{Q}$. Note that the nondimensional temperature, $\tilde{\theta}$ is in the form of a Grashof number:

$$
\operatorname{Gr} \equiv \frac{g \beta D^{3} \Delta T}{\nu^{2}}
$$

Substituting $T_{\infty}^{-1}$ for the coefficient of thermal expansion, $\beta$, and taking the temperature difference to be $T(\mathbf{x})-T_{\infty}$, we recover $\mathrm{Gr}=\tilde{\theta}$. However, we distinguish these parameters because the Grashof number 
typically corresponds to a specific temperature difference. We also have the relation $\operatorname{Ra}=\mathrm{Gr} \operatorname{Pr}$, which relates $\tilde{\theta}$ to the Rayleigh number.

Under the Boussinesq approximation the net buoyancy, $B$ may be expressed

$$
B=\int\left(\rho_{\infty}-\rho\right) g d V=\int\left(\frac{T-T_{\infty}}{T_{\infty}}\right) \rho_{\infty} g d V,
$$

or, nondimensionally:

$$
\frac{B}{\rho_{\infty} g V}=\frac{1}{V} \int\left(\frac{T-T_{\infty}}{T_{\infty}}\right) d V \equiv \theta_{b},
$$

where $\theta_{b}$ denotes the volume-averaged balloon temperature. The net buoyancy can be made nondimensional to yield the scaled temperature:

$$
\frac{B D^{3}}{\rho_{\infty} \nu_{\infty}^{2} V}=\tilde{\theta}_{b}
$$

For a spherical balloon this reduces to:

$$
\tilde{\theta}_{b}=\frac{6 B}{\pi \rho_{\infty} \nu_{\infty}^{2}}
$$

\section{B. Prediction of Net Buoyancy}

Free convection around immersed bodies and within enclosures has been extensively studied. Based on laminar and turbulent boundary layer theory, a local relation between the heat flux at the solid surface, the surface temperature, and the temperature outside the boundary layer is postulated:

$$
\dot{q}^{\prime \prime}=h\left(T_{s}-T_{\infty}\right)
$$

where $h$ is the convective heat transfer coefficient. Provided variation of either the heat flux and or temperature over the surface is known, this relation may be integrated over the surface to obtain:

$$
\dot{Q}=A_{s} h_{\text {avg }}\left(T_{\text {savg }}-T_{\infty}\right)
$$

where the average is over the entire surface. Analytical solutions for simple bodies in laminar flow can be shown to lead to an equation for $h_{\text {avg }}$ of the form:

$$
\mathrm{Nu}=\text { fun (Ra, Pr, geometry, surface conditions), }
$$

where

$$
\mathrm{Nu}=\frac{h_{\text {avg }} L}{k}, \quad \mathrm{Ra}=\frac{g\left(T_{\text {savg }}-T_{\infty}\right) L^{3}}{\nu \alpha},
$$

and $L$ is a characteristic length of the body. "Surface conditions" refers to whether the temperature or heat flux is held constant along the surface (or some more complicated arrangement).

For turbulent flow and for laminar situations where no analytical solution exists, relations of the form of Eq. 14 may still be expected based on dimensional analysis. In those cases, data from many experiments may be used to determine an approximate analytical expression for the functional dependence. In what follows, the subscripts denote the two temperatures used to define the temperature difference in the Rayleigh number and definition of $h_{\text {avg. }}$. The length scale for Ra is taken to be the outer balloon diameter, $D_{o}$ for external convection, the inner diameter $D_{i}$ for internal convection, and the gap distance, $\frac{D_{o}-D_{i}}{2}$ for gap convection.

\section{External convection}

For the external convection, several correlations are available from the literature. Wu and Jones ${ }^{1}$ recommends Campo's ${ }^{3}$ correlation

$$
\mathrm{Nu}_{D o \rightarrow \infty}= \begin{cases}2+0.6 \mathrm{Ra}_{D o \rightarrow \infty}^{0.25} & \mathrm{Ra}<1.5 \times 10^{8} \\ 0.1 \operatorname{Ra}_{D o \rightarrow \infty}^{0.340} & \mathrm{Ra} \geq 1.5 \times 10^{8}\end{cases}
$$


which differs slightly from Churchill, ${ }^{4}$ who reports

$$
\mathrm{Nu}_{D o \rightarrow \infty}= \begin{cases}2+0.461 \mathrm{Ra}_{D o \rightarrow \infty}^{0.25} & \operatorname{Ra}<1 \times 10^{8}, \\ 0.11 \operatorname{Ra}_{D o \rightarrow \infty}^{0.33} & \operatorname{Ra}_{D o \rightarrow \infty} \geq 1.5 \times 10^{8} .\end{cases}
$$

These correlations are developed for the situation of a external convection around a sphere of uniform temperature. In reality, the free convection inside the balloon determines the distribution of temperature and heat flux at the surface, which is non-uniform.

\section{Internal convection}

For internal convection, Carlson and $\operatorname{Horn}^{5}$ use

$$
\mathrm{Nu}_{b \rightarrow s}=\left\{\begin{array}{ll}
2.5\left(2+0.6 \mathrm{Ra}_{b \rightarrow s}^{0.333}\right) & \mathrm{Ra}<1.35 \times 10^{8}, \\
0.325 \mathrm{Ra}_{b \rightarrow s} & \mathrm{Ra} \geq 1.35 \times 10^{8}
\end{array} .\right.
$$

In the definitions of $\mathrm{Nu}$ and $\mathrm{Ra}$ for the internal convection, we interpret the temperature difference as being specified between the bulk volume-averaged temperature within the balloon, $T_{b}$ and the average surface temperature $T_{D i}$.

\section{E. Convection inside the gap}

For double-walled balloons, Jones (personal communication) uses a correlation for gap convection of the form

$$
\mathrm{Nu}_{D i \rightarrow D o}=0.456 \mathrm{Ra}_{D i \rightarrow D o}^{0.226} .
$$

In this formula, the Nusselt number is based on the averaged heat flux over the average surface area of the two spheres, and the length scale is the gap width. A different formula is reported by Raithby and Hollands: ${ }^{6}$

$$
\mathrm{Nu}_{D i \rightarrow D o}=0.617\left(\frac{1-\phi}{\phi\left(1+\phi^{7 / 5}\right)^{5}}\right)^{0.25} \operatorname{Ra}_{D i \rightarrow D o}^{0.25},
$$

where $\phi=\frac{D_{i}}{D_{o}}$. Finally, Teerstra et al. ${ }^{7}$ suggest corrections to the above to appropriately account for the low to mid Rayleigh number regimes. Their formula is:

$$
\mathrm{Nu}^{*}=2 \sqrt{\pi} \frac{1}{1-\phi}+\left(\mathrm{Nu}_{t r}^{-n}+\mathrm{Nu}_{b l}^{-n}\right)^{\frac{1}{n}}
$$

where $n=2$ is suggested and:

$$
\mathrm{Nu}_{t r}=\frac{\sqrt{\frac{2}{\pi}}}{11520}\left(\frac{1-\phi^{3}}{\phi^{2}(\phi+1)}\right) \mathrm{Ra}, \quad \mathrm{Nu}_{b l}=0.528 \frac{\mathrm{Ra}^{0.25}}{\left(1+\phi^{7 / 5}\right)^{5 / 4}} .
$$

Here the asterisk indicates that the length scale chosen for $\mathrm{Nu}$ and $\mathrm{Ra}$ is, in their case, the square root of the inner sphere area $\left(\sqrt{\pi} D_{i}\right)$. Unlike the Raithby and Hollands and Teerstra et al. formulae, Jones's formula does not depend on the ratio of diameters.

\section{F. Combined correlation}

The above correlations may be combined to yield a prediction of the net buoyancy, $B$. As before:

$$
\frac{6 B}{\pi \rho_{\infty} \nu^{2}}=\tilde{\theta}_{b}=\text { fun }(\tilde{Q}) \text {. }
$$

Note that if we base the Nusselt and Rayleigh numbers on $D_{o}$ we have the following relations.

$$
\mathrm{Nu}=\frac{\tilde{Q} \operatorname{Pr}}{\pi \tilde{\theta}}, \quad \operatorname{Ra}=\operatorname{Pr} \tilde{\theta} .
$$




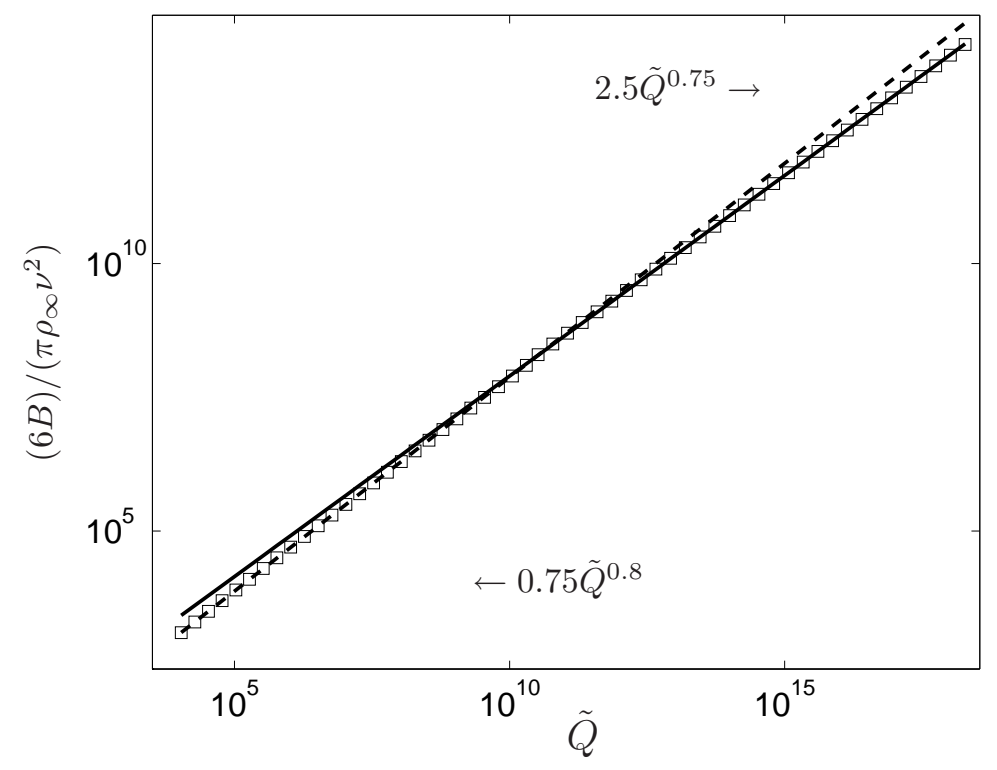

Figure 2. Scaled net buoyancy versus scaled heat input.

The solution procedure is thus as follows. For a specified value of $\tilde{Q}$, we find the temperature change $\tilde{\theta}_{a-b}$ such that the appropriate convection relation between $\mathrm{Nu}_{a-b}$ and $\mathrm{Ra}_{a-b}$ is satisfied. Starting from the outside, we first find the ambient to outer surface temperature change, the change across the gap, and finally the inner surface to balloon interior.

The result of this calculation is shown in Fig. 2 for a single-walled balloon. On the left, we show that prediction for the scaled net buoyancy, $\frac{6 B}{\pi \rho_{\infty} \nu^{2}}$, versus the scaled heat input, $\tilde{Q}$. The transitions from laminar to turbulent correlations is evident at $\tilde{Q} \approx 10^{10}$. However, it is interesting to note that the change in overall exponent for $\tilde{Q}$ is very slight. Based on a best fit to the tabulated values we can propose, approximately:

$$
\frac{6 B}{\pi \rho_{\infty} \nu^{2}}= \begin{cases}0.75 \tilde{Q}^{0.8} & \tilde{Q}<10^{10} \\ 2.5 \tilde{Q}^{0.75} & \tilde{Q}>10^{10}\end{cases}
$$

\section{Computational methodology}

For laminar convection computations, we directly solve (i.e. with no additional turbulence model) the governing equations representing conservation of mass, momentum, and energy (equations 5-7) using a conventional, staggered-mesh, incompressible finite volume scheme on a regular Cartesian mesh. The balloon geometry is modeled using an immersed boundary method, ${ }^{8,9}$ where the boundary conditions at the surface of the balloon (no-slip) are satisfied by adding (regularized) body forces along the surface and determining their strength such that the no-slip boundary condition is enforced. The wall is presumed infinitely thin such that the temperature distribution is continuous through the surface. The outer boundary of the computational domain is prescribed at ambient temperature and zero velocity, but it is placed sufficiently far away that it is verified to have no discernible impact on the flow field or buoyancy.

In the laminar case, we considered spherical, single and double-walled balloons. The source region where heat is added is localized to a small spherical volume along the axis of symmetry and $0.6 D_{o}$ below the center of the balloon. Some tests (not shown here) indicated that the results are not particularly sensitive to source locations below the center of the balloon provided the source region is small compared to the balloon diameter.

The laminar computations were verified by comparing with analytical solutions for (i) transient pure conduction (gravity is switched off) for the given heat source, and (ii) superposed uniform flow over the sphere at a low Reynolds number. Moreover, for the balloon results presented here, several cases were run at progressively finer grid resolutions to check for grid convergence. 
For turbulent calculations the commercial CFD code Fluent ${ }^{10}$ was used together with different Reynolds-Averaged Navier-Stokes turbulence models. The model solves for the (time-averaged) steady state velocity and temperature field. Different RANS models that have one or several tunable parameters are available. For the results presented in Section VI we have used the $k-\varepsilon$ model, ${ }^{11}$ in which the solution of two separate transport equations allow the turbulent velocity and length scales to be independently determined. The constants in the model are kept at their default (factory) settings. To assess the sensitivity to the turbulence model we have, for some particular setups, used the other turbulence models and perturbed the parameter settings in the standard $k-\varepsilon$ mode (this is described in Appendix A).

The turbulent calculations were performed for a sphere-on-cone shaped balloon whose shape was provided in tabular form and is depicted in Fig. 3. Based on its surface area, $S_{b}$, the balloon has an equivalent diameter $D_{o}=\sqrt{\frac{S_{b}}{\pi}}=0.9843 \mathrm{~m}$. The heat source was modeled in an identical fashion as in the laminar case, and was varied over a wide range of values corresponding to nondimensional heat inputs, $\tilde{Q}$ in the range of $10^{12}$ to $2 \times 10^{17}$. Values in the range of $10^{14}$ to $10^{15}$ correspond to the scaled experiments discussed in the next section. The highest values, $2 \times 10^{17}$, correspond to a full-scale Titan Montgolfiere with a $\sim 10 \mathrm{~m}$ diameter and 2000W heat input.

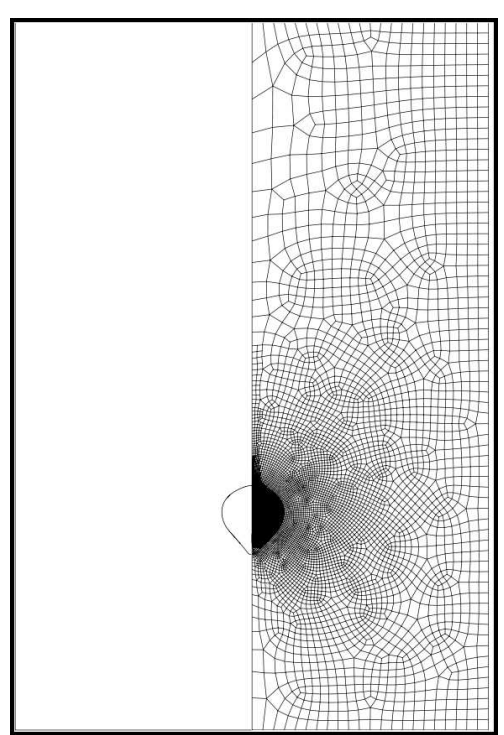

Figure 3. Balloon geometry and base grid for for the Fluent simulations.

\section{Experimental methodology}

Practical data was acquired using the Titan Sky Simulator ${ }^{\mathrm{TM}}$. The simulator is a box 2.5 meters square and 5 meters tall, see Fig. 4. It is highly insulated, see Fig. 4 and the interior is cooled by adding liquid nitrogen, which evaporates filling the interior with nitrogen gas somewhat above its boiling point.

The balloon was flown at various temperatures down to -170C / 103K, see Fig. 4. The resulting thermophysical properties of $N_{2}$ are given in Table 1. While this differs slightly from the target ambient temperature of Titan (which is about 20 degrees colder), it is significant that the balloon was flown in conditions where only a negligible amount of the total heat transfer was by radiation. For balloons flying in terrestrial conditions the majority of heat transfer is by thermal radiation. However radiation varies with the fourth power of absolute temperature. At Titan's low absolute temperature radiation falls dramatically and is of little importance. This balloon was flown at temperatures low enough that radiation was small.

The balloon was heated using a resistance heater. This was inside the balloon but did not touch it at any point. Care was taken to ensure that the heater had a large enough surface area so that almost all heat entered the balloon by convection. The surface temperature of the heater was monitored to confirm that it was cold enough that only a negligible amount of heat was being transferred by thermal radiation. The balloon was suspended by a line from above, which passed through the crown. However it was not attached to the crown but supported the balloon only at the base. In this way the balloon flew as it normally would with the load carried only by the mouth. Fans and interior baffling were used to mix the interior gas to achieve a uniform temperature, while at the same time minimizing wind in the region where the balloon is flying.

Twenty-four thermocouples where installed on the balloon and throughout the Simulator. These measured the internal temperature of the balloon, the temperature of the balloon film at several points and the ambient temperature of the walls and bulk gas. The measurements which are of greatest interests are the temperature differences between the balloon and its surroundings. So the thermocouples were installed in a differential mode, with hot junctions attached to the points of interest and the cold junctions all clamped together to a common temperature reference point, although electrically isolated. This has the advantage that most of the thermocouple wire is copper, reducing the resistance in the long run to the external measuring equipment. 

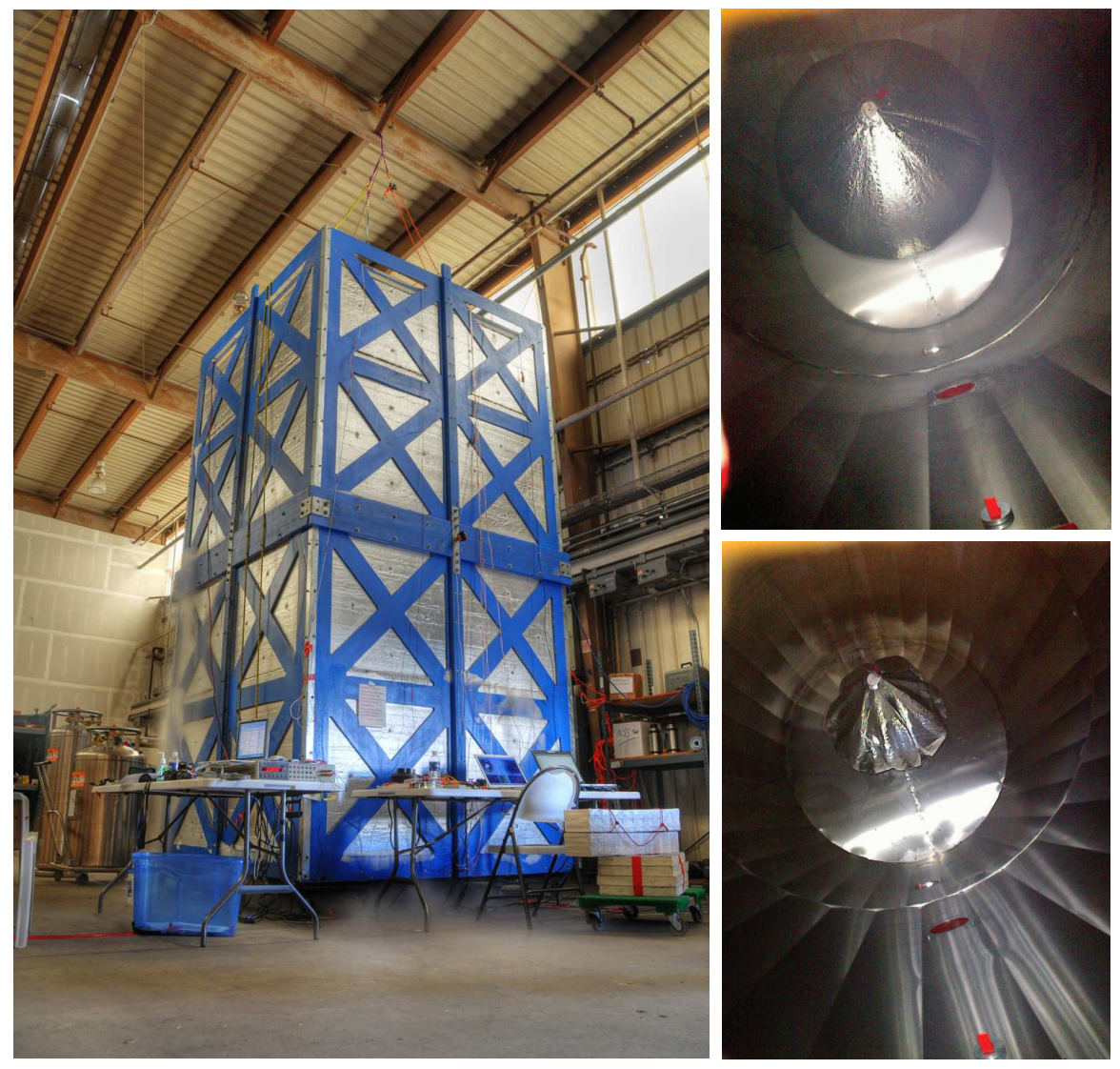

Figure 4. Exterior view of the Titan Sky Simulator ${ }^{\mathrm{TM} 2}$ (left) and view of the balloon inside the simulator before start of experiment (bottom right) and "flying" at low temperature (top right).

\begin{tabular}{|c|c|c|}
\hline$\rho_{\infty}$ & 3.3310 & $\mathrm{~kg} / \mathrm{m}^{3}$ \\
$C_{p}$ & 1068.3 & $\mathrm{~J} / \mathrm{kg} / \mathrm{K}$ \\
$T$ & 103 & $\mathrm{~K}$ \\
$\nu$ & $2.15 \cdot 10^{-6}$ & $\mathrm{~m}^{2} / \mathrm{s}$ \\
$k$ & 0.0102 & $W / \mathrm{m} / \mathrm{K}$ \\
$\operatorname{Pr}$ & 0.811 & - \\
$g$ & 9.82 & $\mathrm{~m} / \mathrm{s}^{2}$ \\
$D_{o} B$ & 0.9843 & $m$ \\
$\dot{Q}$ & 198 & $W$ \\
\hline
\end{tabular}

Table 1. Physical properties for nitrogen at $103 K$ used in the turbulent simulations. 


\section{Laminar convection on single and double-walled balloons}

Results from laminar simulations employing a range of (nondimensional) heat inputs and gap widths are shown in Fig. 5 - 7, where streamlines and temperature contours are drawn at steady state in order to discuss qualitative features of the convective flow.

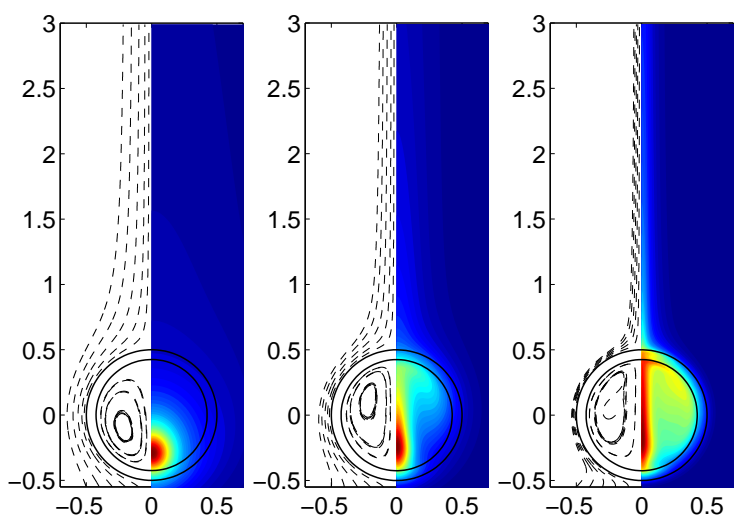

Figure 5. Steady state streamlines (left) and temperature contours (right) for a double-walled balloon with $\phi=0.85$, and heat inputs, from left to right, of $\tilde{Q}=10^{4}, 10^{6}, 15 \cdot 10^{7}$.
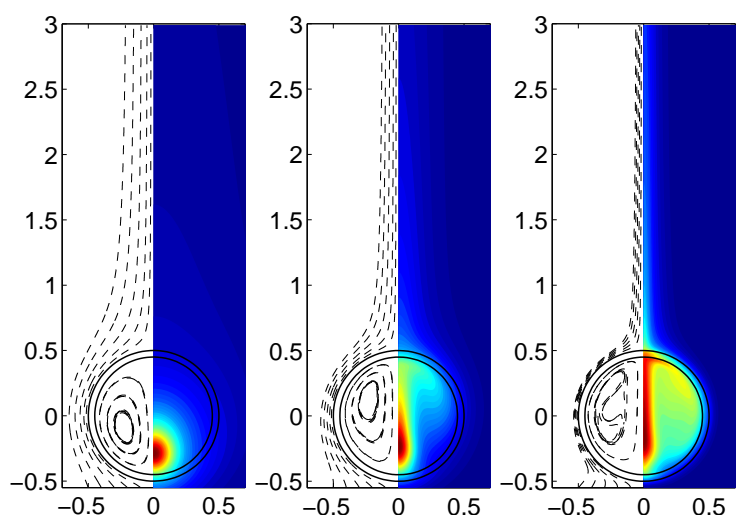

Figure 6. Same as Fig. 5, but with $\phi=0.90$.

For the lowest heat inputs, the flow patterns are substantially different; not much flow is generated and temperature contours are reminiscent of the pure conduction problem. However, as the heat input is increased, a stronger and progressively thinner plume is generated inside the balloon. The temperature around the periphery of the balloon becomes progressively more nonuniform with a strong hot spot at the top of the balloon. The external boundary layer also becomes progressively thinner. Once the plume assumes the more slender shape, its shape remains the same, even when the heat input is increased significantly, see especially Fig. 7. The shape of the plume does not depend significantly on the gap size, but for the largest gap considered, it appears as if the temperature is more evenly distributed, especially for stronger heat inputs.

In Fig. 8 the results from the laminar simulations are presented in terms of Rayleigh and Nusselt numbers at steady state, corresponding to the temperature difference between the internal volume-averaged temperature and the inside-sphere surface averaged value. These data are compared to the internal heat transfer correlation given by Eq. 18. At very low Rayleigh numbers the simulations collapse onto a constant Nusselt number, corresponding to the conduction limit. Over the range of Rayleigh numbers where a thin laminar convection boundary layer, there is progressively better agreement with the turbulent correlation. It is unclear why the laminar correlation performs so poorly, or why the results agree better with the turbulent one. We can also observe that the results do not collapse to a single correlation independent of the gap thickness. 

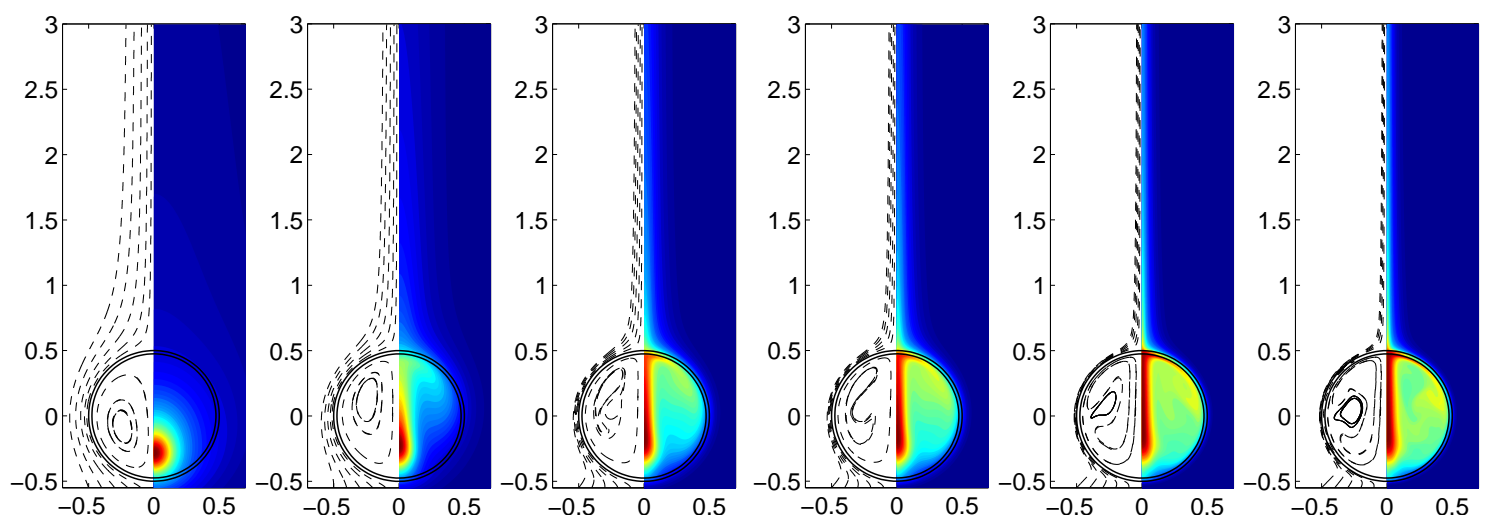

Figure 7. Same as Fig. 5 , but with $\phi=0.95$, and with 6 values of $\tilde{Q}: 10^{4}, 10^{6}, 6 \cdot 10^{6}, 15 \cdot 10^{7}, 4 \cdot 10^{9}, 8 \cdot 10^{9}$.

This indicates that the internal temperature distribution is changed as the gap thickness is changed, and this provides some guidance as to the sensitivity of the correlations to the details of the temperature distribution (for the laminar case).

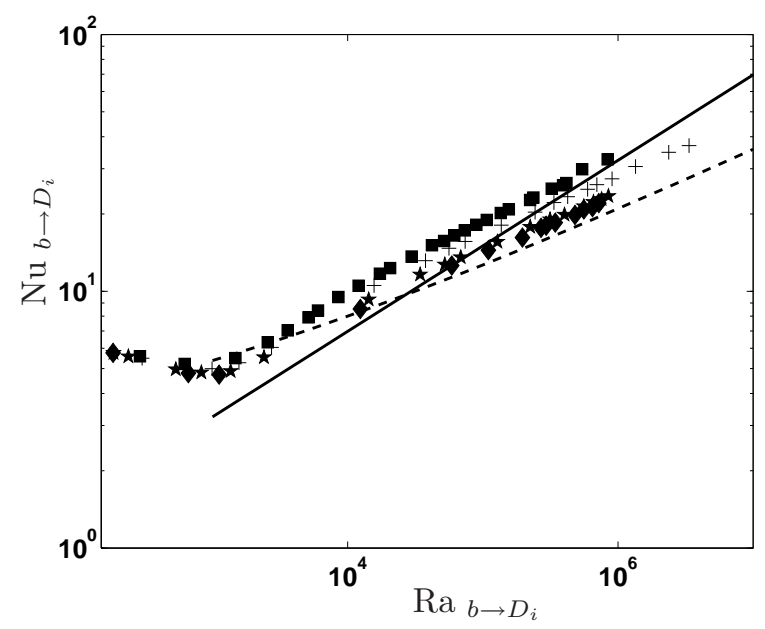

Figure 8. Comparison between steady-state laminar simulation results and the internal convection correlation (19). $(\boldsymbol{\square}, \phi=1 ;+, \phi=0.95 ; \diamond, \phi=0.90 ; \star, \phi=0.85)$. The dashed line represents the laminar (low Ra) correlation while the solid line represents the turbulent (high Ra) one.

In Fig. 9 convection in the gap is considered. The correlation suggested by Teerstra et al. ${ }^{7}$ and the correlation represented by (19) are compared to the simulation results. The simulations result in relatively low gap Rayleigh numbers, and appear to be closer to the conduction limit that the laminar boundary layer regime implied by Eq. 19. In the pure conduction limit $(\mathrm{Ra} \rightarrow 0)$, the simulation results agree with Eq. 21, but fail to be captured by the correlation as convection begins to occur. A possible explanation of the discrepancy could again be the non-uniform temperature distribution.

In Fig. 10 the external correlation (16) is compared to simulation results for double- and the single-wall balloon cases. Despite the fact that the correlation is derived for uniformly heated sphere, the simulation data (with nonuniform temperature) agree relatively well with the correlation, especially at the higher values of Ra considered.

In Fig. 11 all results from the laminar simulations are collected and expressed in terms of the nondimensionalized heat input and buoyancy. The solid black line is the correlation (Eq. 23) for the single-walled balloon. As can be seen, the simulation data for the single walled balloon line up relatively well with the overall correlation, despite some differences for the individual internal and external convection coefficients. The significantly increased buoyancy (and thus payload) in double-walled designs is also apparent, although, 


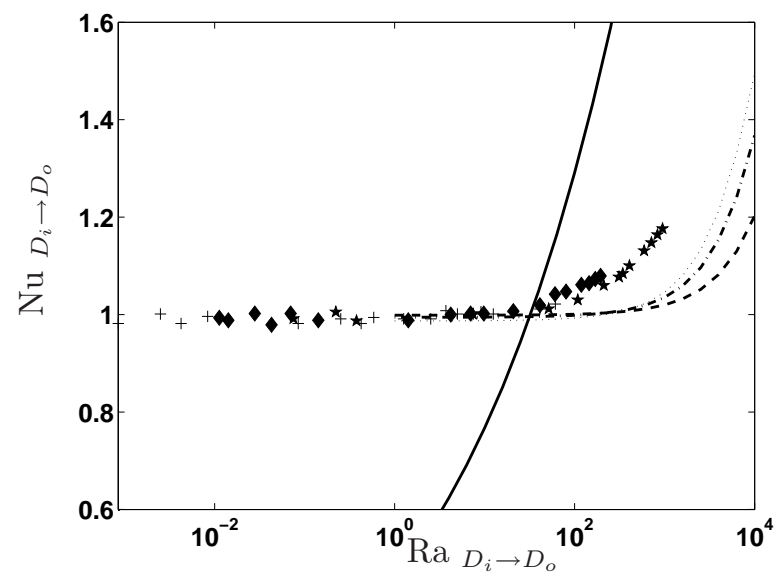

Figure 9. Comparison between steady-state laminar simulation results and correlations for gap convection. $(\square, \phi=1 ;+, \phi=0.95 ; \diamond, \phi=0.90 ; \star, \phi=0.85)$. The solid line to the right is the correlation (19) and the dashed, dash-dotted and the dotted lines are curves describing the correlation (21) with $\phi=0.95,0.90,0.85$.

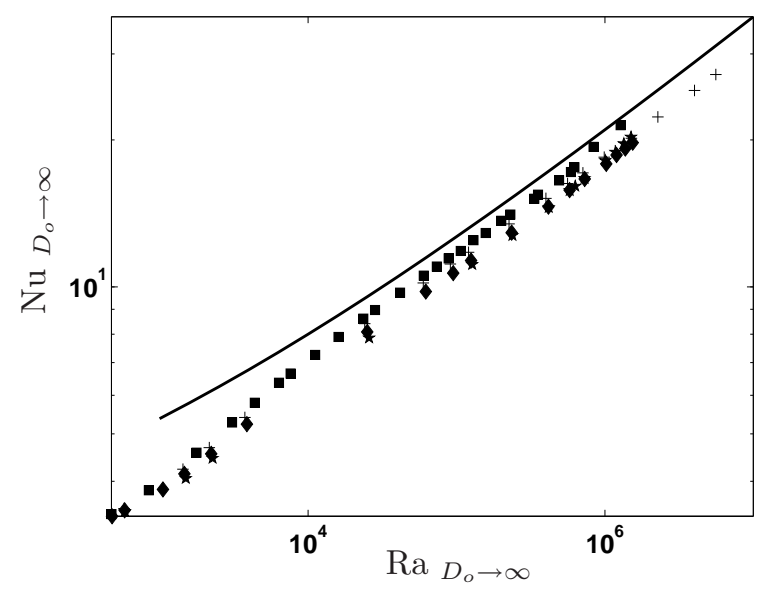

Figure 10. Comparison between steady-state laminar simulation results and correlations. ( $\boldsymbol{\square}, \phi=1 ;+, \phi=$ $0.95 ; \diamond, \phi=0.90 ; \star, \phi=0.85)$. The solid line to the left is the correlation (16). The solid line to the right is the combined correlation computed ad described in Section F. The solid, dashed, dash-dotted and the dotted lines are for the cases with $\phi=1.0,0.95,0.90,0.85$.

owing to the relatively poor performance for the gap convection prevents the theory from giving good predictions for the double-walled case. form

Given the double-walled simulation results, we may offer a least square fit to a power-law model of the

$$
\theta_{b}=f(\tilde{Q})=\chi_{1} \tilde{Q}^{\chi_{2}} .
$$

The values are given in Table 2.

\section{Turbulent convection: comparison of experiment, computation and theory}

In this section, we compare results from turbulent simulations using the Fluent model for single-walled balloons to the experiments performed in the Titan Sky Simulator ${ }^{\mathrm{TM}}$ and the empirical correlation (23). In Table 3 heat input and measured lift values (in SI units) from experiments are reported. Using the thermo-physical properties of nitrogen (table 1) we may convert the heat input and lift to nondimensional 


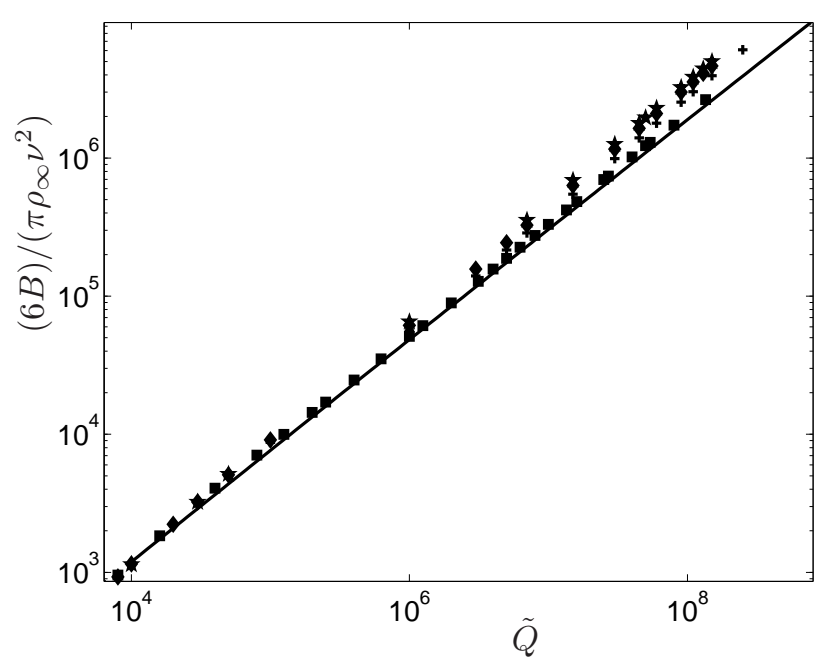

Figure 11. Comparison between laminar simulation results and correlations. Displayed is the non-dimensional buoyancy against non-dimensionalized heat input. The $\mathbf{\square},+, \diamond$ and $\star$ represent computational results for balloons with $\phi=1,0.95,0.90,0.85$. The solid line to the left is the predicted buoyancy using equation (23).

\begin{tabular}{|c|c|c|c|c|}
\hline$\delta$ & 1.0 & 0.95 & 0.90 & 0.85 \\
\hline \hline$\chi_{2}$ & 0.752 & 0.840 & 0.858 & 0.867 \\
\hline$\chi_{1}$ & 0.806 & 0.530 & 0.444 & 0.412 \\
\hline
\end{tabular}

Table 2. Values of the parameters in the model (24) for laminar convection in double-walled balloons.

values, $\tilde{\theta}$ and $\tilde{Q}$, respectively, and plot them against the correlation in Fig. 12. It should be noted that the different ambient temperatures lead to different values of nondimensional heat input, even when the physical heat input is held constant. Also plotted in the figure are the Fluent simulation results computed using the standard $k-\varepsilon$ RANS turbulence model. In Table 3 the relative deviations (in \%) from the correlation are also reported.

The experimental and computational results line up well with the empirical correlation. This provides, for the first time, a direct confirmation of the turbulent internal and external heat transfer correlations leading to Eq. 23 and validates their use for system-level models for the Titan Montgolfiere. The data generally lie above the theoretical predictions (more buoyancy), showing that the correlations are conservative and that a balloon designed using them could be equipped with larger payload than predicted. The agreement between simulation and theory is especially close, showing less than $10 \%$ differences over a large range of heat inputs all the way to projected full-scale values. There is more scatter in the experimental results, especially for two outliers, and it would be useful to follow up these preliminary experiments to obtain a better understanding of repeatability and uncertainty in the data.

Finally, the numerical values of the computational predictions are reported in Table 4 . The highest value for $\tilde{Q}$ used in the computations is equivalent to that of a 9-meter balloon flying in nitrogen at 83K on Titan with a $2000 \mathrm{~W}$ heat source. If the computational result (the rightmost data point) is converted to dimensional lift we find that such a balloon is predicted to yield a lift of $176 \mathrm{~kg}$ on Titan (or $25 \mathrm{~kg}$ on Earth).

Table 4. Computed non-dimensional buoyancy from simulations with Fluent. Values in parenthesis are relative deviation (in \%) from the correlation.

\begin{tabular}{||l||c|c|c|c|c|c||}
\hline$\tilde{Q} / 10^{17}$ & 2.61 & 1.30 & 0.0522 & 0.0496 & 0.0496 & 0.0109 \\
\hline$\left(6 B / \pi \rho_{\infty} \nu_{\infty}^{2}\right) / 10^{13}$ & $3.05(6.8)$ & $1.83(7.5)$ & $0.156(1.5)$ & $0.151(2.0)$ & $0.0485(1.4)$ & $0.0255(5.4)$ \\
\hline
\end{tabular}




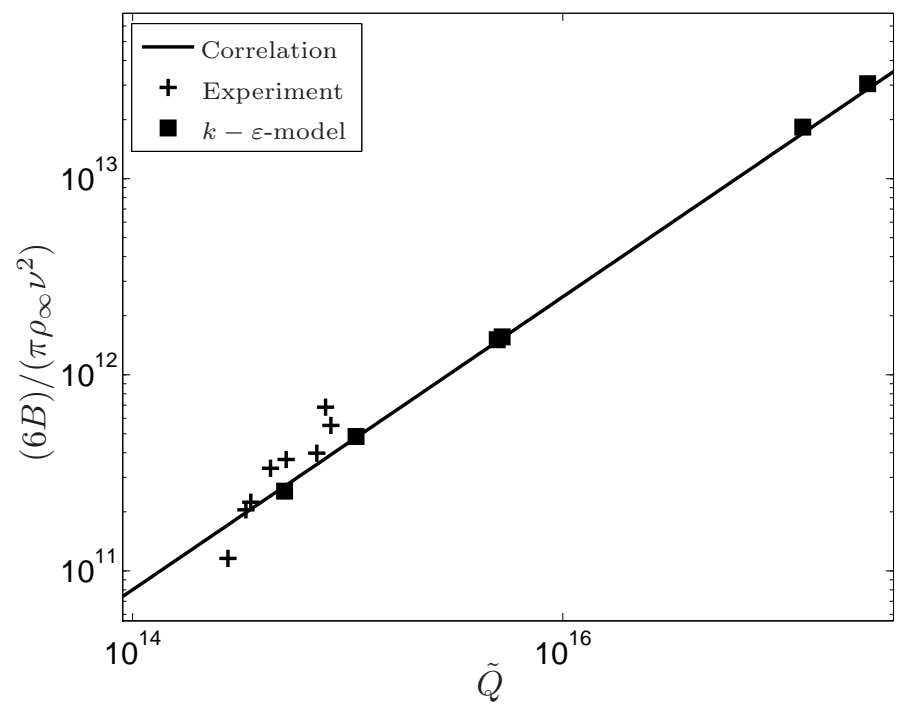

Figure 12. Fluent turbulent simulation results, experiments and correlation. Displayed is the non-dimensional buoyancy against non-dimensionalized heat input for the $k-\varepsilon$ model (black squares), experimental data (black + signs) and the combined correlation (23) (black line). The values at the right boundary of the plot correspond to a full-sized balloon.

\section{Summary and future work}

Computational models to predict the natural convection and buoyancy of idealized hot air balloons have been developed and compared to theoretical/empirical heat transfer correlations and experimental data from the Titan Sky Simulator ${ }^{\mathrm{TM}}$ cryogenic facility. Separate algorithms were used for the laminar and turbulent regimes, and the latter utilized standard Reynolds-averaged turbulence models.

Since the laminar simulations involve no modeling uncertainties beyond the idealization of the heat source, they may be used to establish the detailed credentials of the heat transfer correlations. While overall predictions for the single-walled balloons were satisfactory, gap convection correlations

Table 3. Experimental. Values in parenthesis are relative deviations (in \%) from the correlation. did not provide satisfactory agreement with the sim-

\begin{tabular}{|l|l|l|}
\hline$Q[\mathrm{w}]$ & $T_{\infty}[\mathrm{K}]$ & $g B[\mathrm{Kg}]$ \\
\hline \hline 198 & 103 & $0.304(35)$ \\
422 & 158 & $0.327(14)$ \\
422 & 136 & $0.453(41)$ \\
422 & 144 & $0.561(83)$ \\
195 & 120 & $0.274(38)$ \\
195 & 148 & $0.184(9)$ \\
195 & 156 & $0.168(3)$ \\
195 & 189 & $0.095(33)$ \\
\hline
\end{tabular}
ulations.

The turbulent (single-walled) results were in good agreement with experiments and were especially close (within 10\%) to the empirical correlation for a broad range of heat inputs, corresponding from model to full-scale balloons being design for exploration of Titan.

Our future work will extend the turbulent models for double-walled balloon designs, mixed forced/natural convection around ascending and descending balloons, and to examine heat losses and changes in buoyancy associated with vents and other geometrical variations of the balloon.

\section{A. Appendix}

To asses the sensitivity of the computed turbulent correlations we used different RANS models (SpalartAlamaras, $k-\varepsilon, k-\omega)$ with their respective "factory setups" to predict the buoyancy. The results in terms of computed non-dimensional buoyancy from this study are reported in Table 4 and Fig. 13. As can be seen in Fig. 13, the computational results line up along the correlation for all models except the Spalart-Alamaras 
model which predicts a slightly higher buoyancy. In Fig. 13 the non-dimensional buoyancy computed from data from the Titan Sky Simulator ${ }^{\mathrm{TM}}$ are also plotted. As reported above, the experimental data also fall in the vicinity of the correlation but with more spread than the computational models. Finally, for the $k-\varepsilon$

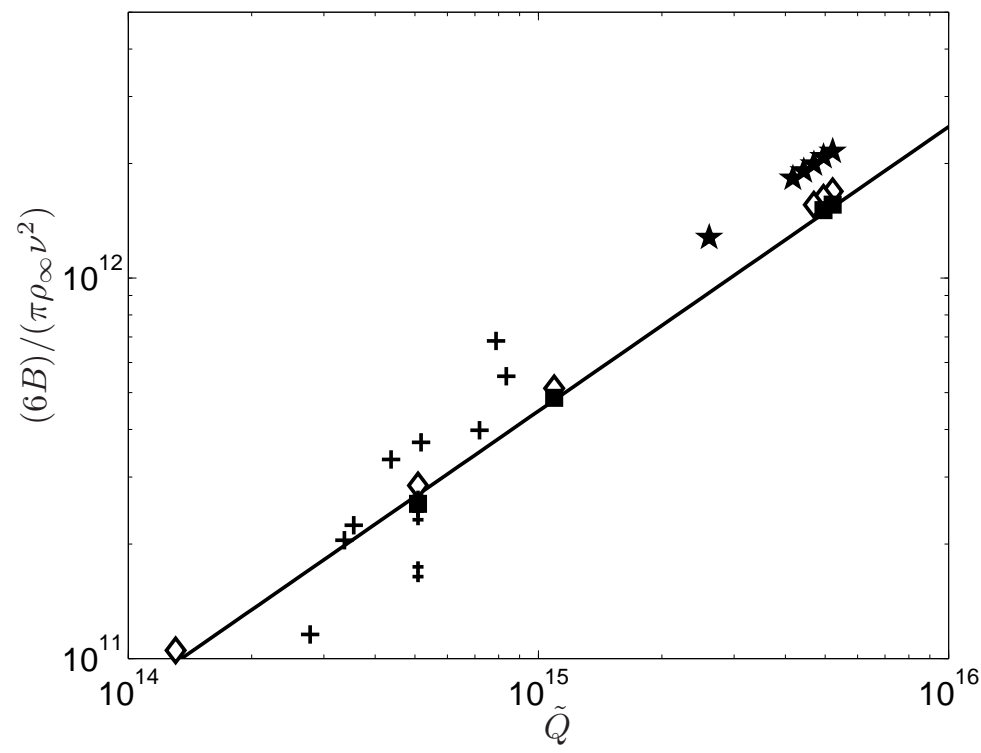

Figure 13. Non-dimensional buoyancy against non-dimensionalized heat input for $k$ - $\varepsilon$ model (black squares), $k-\omega$ model, (diamonds), Spalart-Alamaras model (pentagrams) and the correlation (black line). The larger black + are experimental results and the smaller are the results from Table 5.

model we perturbed the adjustable parameters $C_{\mu}, C_{1}-\varepsilon$ and $C_{2}-\varepsilon$ up or down $10 \%$ relative their factory settings values. We also used the two other $k-\varepsilon$ models "RNG", and "Realizable" to predict the buoyancy. The results, which can be found in Table 5 , show that the output is relatively insensitive (with the exception of perturbations to $C_{2}-\varepsilon$ ) to perturbations.

Table 5. Perturbation of $k-\varepsilon$ at $\tilde{Q}=509 \times 10^{12}$.

\begin{tabular}{|c|c|c|c|c|c|c|c|c|}
\hline$k-\varepsilon$ model & $C_{\mu}$ & $C_{1}-\varepsilon$ & $C_{2}-\varepsilon$ & TKE Pr & TKR Pr & Energy Pr & $\left(6 B / \pi \rho_{\infty} \nu_{\infty}^{2}\right) / 10^{9}$ & $\%$ diff \\
\hline \hline STD & 0.09 & 1.44 & 1.92 & 1 & 1.3 & 0.85 & 255.1 & 5.4 \\
STD & 0.09 & 1.44 & 2.09 & 1 & 1.3 & 0.85 & 174.2 & 35.4 \\
STD & 0.09 & 1.44 & 1.71 & 1 & 1.3 & 0.85 & 164.2 & 39.1 \\
STD & 0.081 & 1.44 & 1.92 & 1 & 1.3 & 0.85 & 244.5 & 9.4 \\
STD (visc. heat) & 0.09 & 1.44 & 1.92 & 1 & 1.3 & 0.85 & 243.9 & 9.6 \\
\hline RNG & & & & & & & 267.1 & 0.98 \\
Realizable & & & & & & & 244.1 & 9.5 \\
\hline
\end{tabular}

\section{References}

\footnotetext{
${ }^{1}$ Jones, J. and Wu., J.-J., "Performance model for reversible fluid balloons," AIAA Paper 95-1608-CP, 1995.

${ }^{2}$ Nott, J. and Rand, J., "The Titan Sky Simulator ${ }^{\text {TM }}$ Test Facility," AIAA-2007-2625, AIAA Balloon Systems Conference, Williamsburg, VA, May 21-24, 2007.

${ }^{3}$ Campo, A., "Correlation equation for laminar and turbulent natural-convection from spheres," Warme Und Stoffubertragung-Thermo and Fluid Dynamics, Vol. 13, No. 1-2, 1980, pp. 93-96.

${ }^{4}$ Churchill, S., Heat Exchanger Design Handbook, chap. Free convection around immersed bodies, Hemisphere, 1983.

${ }^{5}$ Horn, W. J., "New thermal and trajectory model for high-altitude balloons," Journal of Aircraft, Vol. 20, No. 6, 1983, pp. 500-507.
} 
${ }^{6}$ Raithby, G. D. and Hollands, K. G. T., "A general method of obtaining approximate solutions to laminar and turbulent free convection problems," Advances in heat transfer. Volume 11. (A76-17076 05-34) New York, Academic Press, Inc., 1975, p. 265-315., Vol. 11, 1975, pp. 265-315.

${ }^{7}$ Teertstra, P. M., Yovanovich, M. M., and Culham, J. R., "Analytical Modeling of Natural Convection in Concentric Spherical Enclosures," Journal of Thermophysics and Heat Transfer, Vol. 20, No. 2, 2006, pp. 297-304.

${ }^{8}$ Taira, K. and Colonius, T., "The immersed boundary method: A projection approach," Journal of Computational Physics, Vol. 225, No. 2, AUG 10 2007, pp. 2118-2137.

${ }^{9}$ Colonius, T. and Taira, K., "A fast immersed boundary method using a nullspace approach and multi-domain far-field boundary conditions," Computer Methods in Applied Mechanics and Engineering, Vol. 197, April 2008, pp. $2131-2146$.

${ }^{10}$ Fluent Inc., http://www.fluent.com/.

${ }^{11}$ Launder, B. E. and Spalding, D. B., Lectures in mathematical models of turbulence, Academic Press, London, 1972. 